\title{
Elucidating Cathodic Corrosion Mechanisms with Operando Electrochemical Liquid-Cell STEM in Multiple Dimensions
}

Yao Yang ${ }^{1}$, Yu-Tsun $\mathrm{Shao}^{2}$, Xinyao Lu ${ }^{1}$, Héctor Abruña ${ }^{3}$ and David Muller ${ }^{4}$

${ }^{1}$ Department of Chemistry and Chemical Biology, Cornell University, Ithaca, New York, United States,

${ }^{2}$ School of Applied and Engineering Physics, Cornell University, Ithaca, NY, USA, Ithac, New York, United States, ${ }^{3}$ Department of Chemistry and Chemical Biology, Cornell University, United States,

${ }^{4}$ School of Applied and Engineering Physics, Cornell University, Ithaca, NY, USA, Ithaca, New York, United States

Cathodic corrosion is an enigmatic electrochemical process that corrodes metallic electrodes under sufficiently reducing potentials. First discovered by Fritz Haber in the $19^{\text {th }}$ century, ${ }^{1-3}$ the dynamic cathodic corrosion mechanisms remain elusive, due to the lack of operando/in situ microscopic methods for probing electrified solid-liquid-gas interfaces. ${ }^{4}$ These poorly-understood mechanisms also have modern relevance, as they underlie the structural evolution of nanosized electrocatalysts during many electrochemical reactions under reducing potentials, in particular, $\mathrm{CO}_{2}$ and $\mathrm{N}_{2}$ reduction for their potential to lower carbon emission and produce valuable chemicals. 5,6

Here, we employed operando electrochemical liquid-cell STEM (EC-STEM) to reveal that cathodic corrosion led to a significantly higher level of structural destruction for $\mathrm{Au}$ nanocubes than bulk $\mathrm{Pt}$ electrodes, accompanied by the unexpected formation of Au-Pt alloy nanostructures, which are thermodynamically immiscible in the Au-Pt phase diagram. Dynamic evolution in morphology, composition, and crystallographic information was retrieved by multidimensional STEM techniques, namely 2D-STEM imaging, 3D STEM-EDX elemental mapping, and 4D-STEM diffraction imaging. We designed a strategy to enable operando, in situ and ex situ EC-STEM operation in the same cell, and at identical locations to enable reliable electrochemical measurements of cathodic corrosion and simultaneously probe real-time interfacial changes across multiple scales (Figs. 1a,b) by generating $\mathrm{H}_{2}$ bubbles to reversibly control the liquid thickness.

Operando EC-STEM was used to track the morphological changes in a thick electrolyte under similar conditions to standard electrochemical measurements (Fig. 2). The nucleation and formation of $\mathrm{H}_{2}$ bubble were evidenced by cyclic voltammetry $(\mathrm{CV})$, where well-defined redox peaks progressively increased as the potential limits decreased from -1.0 to $-1.8 \mathrm{~V}$, followed by a rapid decay to nearly zero at $-2.0 \mathrm{~V}$ vs. Pt (Figs. 2a,b). A native thinner liquid layer, created by the $\mathrm{H}_{2}$ bubble, leads to a significantly improved spatial resolution (Fig. 2c). Operando EC-STEM images in Figs. 2d-f show the agglomeration of nanoparticle (NP) clusters ( 100 nm, white boxes) and generation of new Au-Pt alloy NPs $(\sim 30 \mathrm{~nm}$, red arrows and green boxes).

The thinner liquid made in situ STEM-EDX and 4D-STEM diffraction imaging possible. STEM-EDX maps show the transformation from Au nanocubes near the bulk Pt electrode (Fig. 1c) to Au-rich alloy NPs ( 30 nm, Au/Pt 5:1) and Au-Pt alloy nanostructures (Au/Pt 1:1) on the Au-rich ribbon ( 100 nm) (Fig. 1d). In situ scanning electron nanobeam diffraction (SEND), using an electron microscopy pixel array detector (EMPAD), provides complementary information on crystal structures besides morphology 
and composition. ${ }^{7-10}$ Virtual bright field $(\mathrm{BF})$ image and crystallographic orientation map, obtained by the SEND, show the polycrystalline nature of NP clusters and mossy structures (Figs. 1e,f). To resolve atomic-scale lattice structures, $\mathrm{H}_{2}$ gas was flowed to remove the liquid completely, while preserving the corroded electrode at the same locations in a reducing environment. Ex situ STEM images of Au5 $\mathrm{Pt}$ alloy NPs in $\mathrm{H}_{2}$ gas cell configuration clearly exhibit the "nano-spikes" features on the polycrystalline particle surface (Fig. 1g) and the atomic structure of an fcc lattice oriented near the [110] zone axis (Fig. 1h).

In summary, this work shows higher cathodic corrosion kinetics for nanocrystals than bulk electrodes, and demonstrate how operando EC-STEM provides a platform for microscopic investigation at electrodeelectrolyte interfaces. 11

a
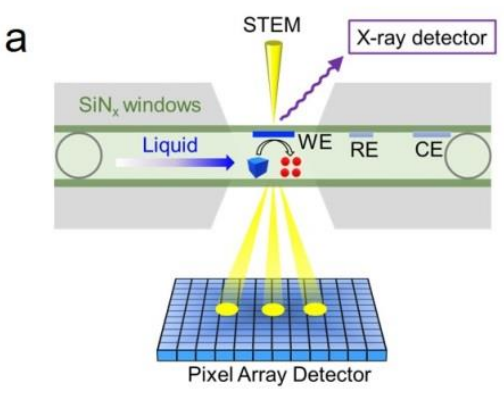

$\mathrm{b}$

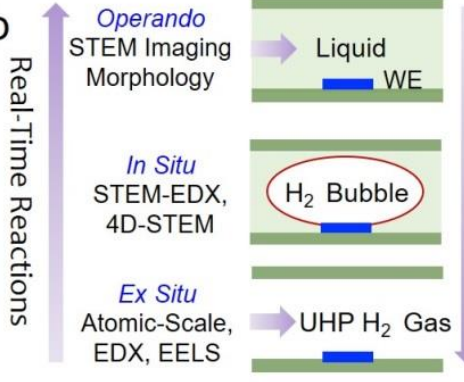

IIn Situ Thin Liquid
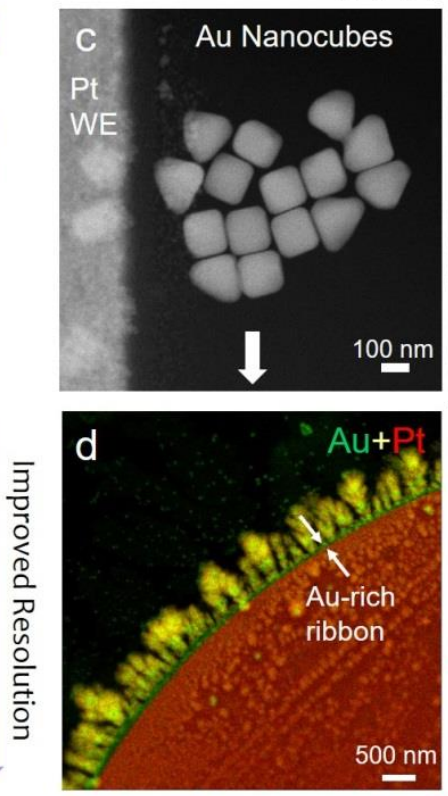
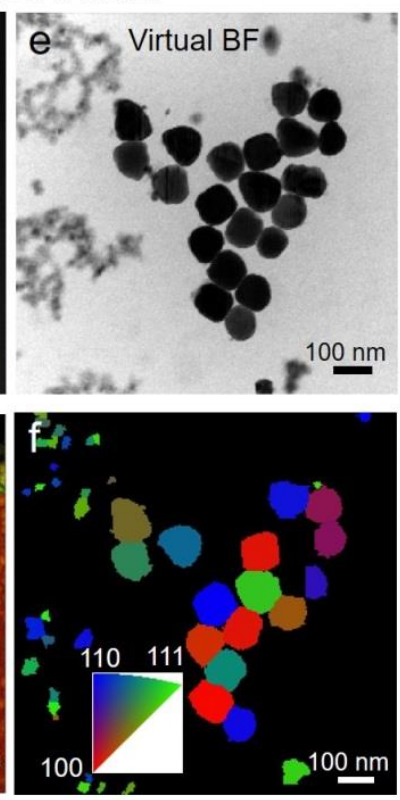

Ex Situ $\mathrm{H}_{2}$ Gas Cell I
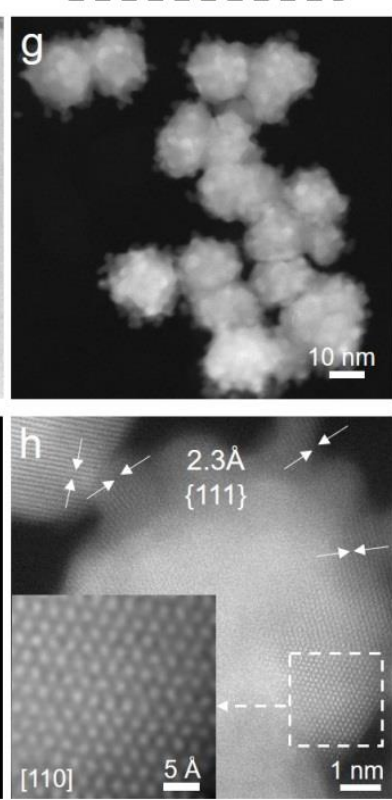

Figure 1. Operando/in situ electrochemical liquid-cell STEM studies of cathodic corrosion. (a) Schematic of EC-STEM with working, reference and counter electrodes (WE, RE and CE), and EDX and 4D-STEM capability in liquid. (b) Overview of methodology of operando/in situ/ex situ EC-STEM. (c) High-angle annular dark-field (HAADF) STEM image of pristine Au nanocubes near the Pt WE in liquid. (d) In situ thin-liquid STEM-EDX mapping of heterogenous Au-Pt alloy nanostructures after corrosion. (e-f) In situ thin-liquid BF-STEM image and SEND-based crystallographic orientation map of corroded NP clusters and mossy structures, where the color represents the superposition of three basal planes in the inverse pole figure. (g-h) Ex situ atomic-scale HAADF-STEM image of Au5Pt alloy NPs in ultrahigh purity (UHP) $\mathrm{H} 2$ gas cell. 

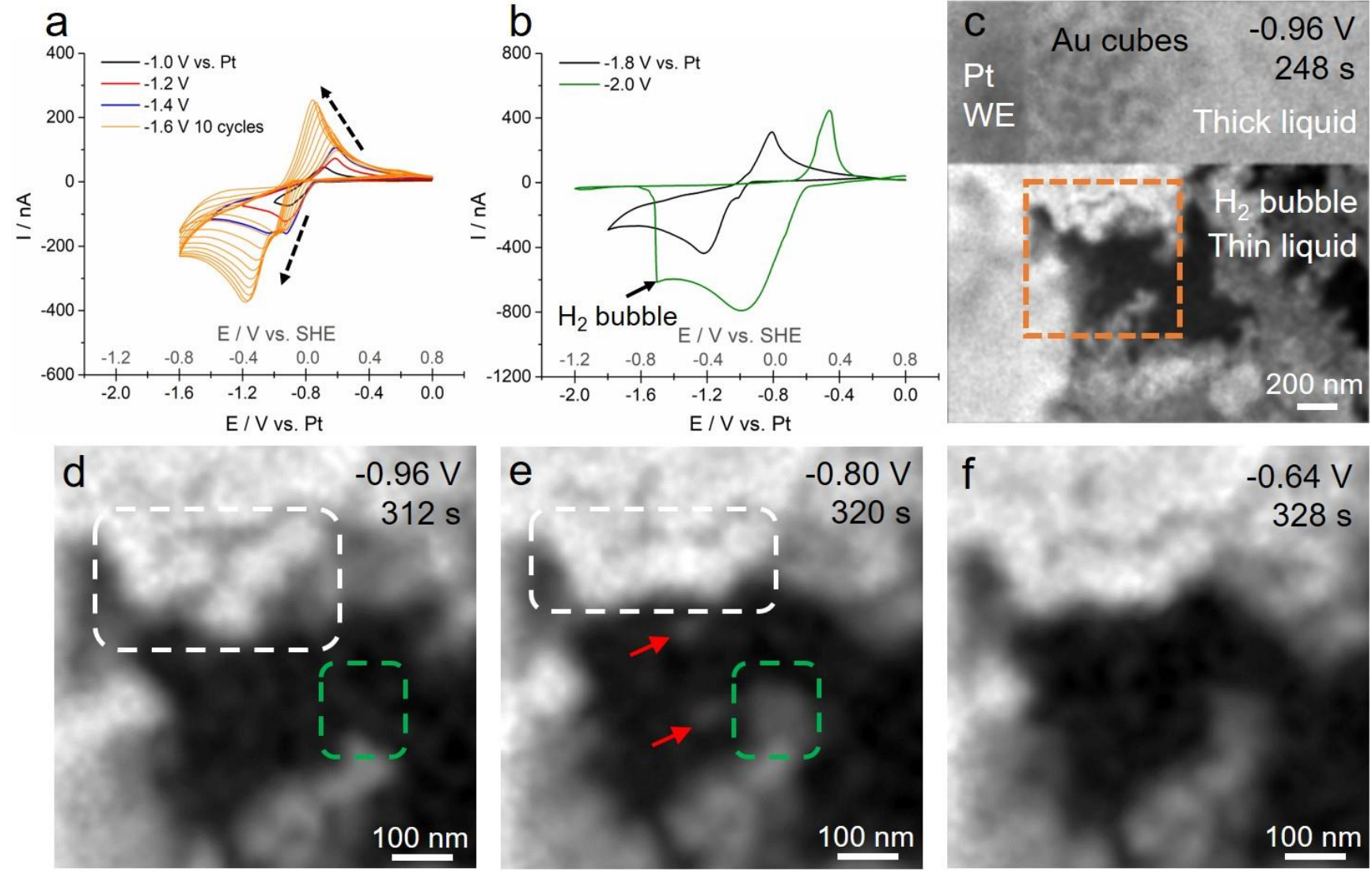

Figure 2. Figure 2 Operando EC-STEM studies of dynamic morphological changes during cathodic corrosion. (a) CV profiles of Au nanocubes on the Pt WE in EC-STEM in a regular thick electrolyte (500$800 \mathrm{~nm}$ ). 10 continuous cycles from -1.6 to $0 \mathrm{~V}$ (orange profiles) showing the process of accumulating soluble $\mathrm{H} 2$. (b) $\mathrm{CV}$ profiles showing a sharp drop in reduction current as the lower limit reached $-2.0 \mathrm{~V}$, indicating the formation of a $\mathrm{H} 2$ bubble. (c) Operando EC-STEM image of the thin liquid layer after the $\mathrm{H} 2$ bubble formation. (d-f) Three continuous STEM image frames showing the dynamic morphological changes during cathodic corrosion.

References

1. F. Haber, Z. Anorg. Chem. 16 438-449 (1898).

2. H. Salzberg, J. Electrochem. Soc. 105, 64-66 (1958).

3. T. Hersbach, A. Yanson, M. Koper, Nat. Commun. 7, 12653 (2016).

4. Y. Yang, D. Muller, H. Abruña, et al. ACS Catal. 11, 1136-1178 (2021).

5. Y. Li, P. Yang, et al. Proc. Natl. Acad. Sci. U. S. A. 117, 9194-9201 (2020).

6. J. Chen, et al. Science 360, 873 (2018).

7. J. M. Zuo, J. Tao, Scanning Electron Nanodiffraction and Diffraction Imaging. (Springer, NY 2011).

8. C. Ophus, Micro. Microanal. 25, 563-582 (2020).

9. M. Tate, D. Muller, S. Gruner, et al. Micro. Microanal. 22, 237-249 (2016).

10. Y. Jiang, Z. Chen, D. Muller, et al. Nature 559, 343-349 (2018).

11. This work was supported by the Center for Alkaline-Based Energy Solutions, an Energy Frontier Research Center program supported by the U.S. Department of Energy, under Grant DE-SC0019445. This work made use of TEM facilities of the CCMR, supported by NSF (DMR-1719875). 